\title{
Erratum to: Comment on "On the general relativistic framework of the Sagnac effect” EPJC 79:187
}

\author{
H. Ramezani-Avala ${ }^{\mathrm{a}}$ \\ Department of Physics, University of Gonabad, Gonabad, Iran
}

Published online: 3 August 2020

(C) Società Italiana di Fisica and Springer-Verlag GmbH Germany, part of Springer Nature 2020

\author{
Erratum to: Eur. Phys. J. Plus \\ https://doi.org/10.1140/epjp/s13360-020-00246-6
}

The original version of this article containes a mistake. In the second line of Comment 2 , it should read: "It is necessary to note that Schwarzschild metric is static while the rotating metric (which is the subject of discussion) is stationary." 